\title{
Dietary patterns and cardiovascular disease-related risks in Chinese older adults
}

\author{
Jing Sun ${ }^{1}$, Nicholas Buys ${ }^{1}$ and Shuying Shen ${ }^{2}$ \\ ' School of Public Health, Griffith Health Institute, Griffith University, Gold Coast, QLD, Australia \\ ${ }^{2}$ Chang Shu Health Inspection Institute, Chang Shu, China
}

\section{Edited by:}

André Amaral, Imperial College

London, UK

Reviewed by:

Charles E. White, Charles E. White's Biostatistical Consulting, LLC, USA Terri Kang Johnson, Food and Drug Administration, USA

Leonardo Roever, University of São

Paulo, Brazil; Federal University of Uberlândia, Brazil

Arthur Eumann Mesas, Universidade Estadual de Londrina, Brazil

*Correspondence:

Jing Sun, Griffith Health Institute, Griffith University, Gold Coast

Campus, Parkland, Gold Coast, QLD Q4222, Australia

e-mail:j.sun@griffith.edu.au
Studies of Western populations demonstrate a relationship between dietary patterns and cardiovascular-related risk factors. Similar research regarding Chinese populations is limited. This study explored the dietary patterns of Chinese older adults and their association with cardiovascular-related risk factors, including hypertension, obesity, and metabolic syndrome. Data were collected using a 34-item Chinese food frequency questionnaire from 750 randomly selected older adults aged 50-88 who participated in the study in 2012. Factor analysis revealed four dietary patterns: a "traditional food pattern," consisting of vegetable, fruit, rice, pork, and fish; a "fast and processed food pattern" consisting of fast or processed food products, sugar, and confectionery; a "soybean, grain, and flour food pattern"; and a "dairy, animal liver, and other animal food pattern." These patterns explained $17.48,9.52,5.51$, and $4.80 \%$ of the variances in food intake, respectively. This study suggests that specific dietary patterns are evident in Chinese older adults. Moderate intake of "traditional Chinese food" is associated with decreased blood pressure and cholesterol level. A dietary pattern rich in soybeans, grains, potatoes, and flour is associated with reduced metabolic factors including reduced triglycerides, fasting glucose, waist circumference, and waist-hip ratio, and a high level of dairy, animal liver, and other animal intake food pattern is associated with increased level of Body Mass Index. In conclusion, this study revealed identifiable dietary patterns among Chinese older adults that are significantly related to blood pressure and metabolic biomarkers. Further study using prospective cohort or intervention study should be used to confirm the association between dietary patterns and blood pressure and metabolic factors.

\section{Keywords: dietary pattern, cardiovascular disease, hypertension, obesity, metabolic syndrome}

\section{INTRODUCTION}

The prevalence of hypertension is increasing and constitutes a major public health issue in China (1). According to the 2002 Chinese national health survey, $28.6 \%$ of the population aged 35 or above has hypertension, with the percentage increasing to $48.8 \%$ for those aged 65 or above (2). Hypertension is associated with leading causes of morbidity and mortality, including metabolic syndrome, diabetes, stroke, heart attack, and kidney failure (3). There is substantial evidence that individuals with different food intake patterns and exercise levels have different levels of cardiovascular risk factors such as high blood pressure, high levels of serum cholesterols, and low-density lipoprotein (LDL)-cholesterol (4-6). For example, people who exercise and consume healthy food have a relatively low risk of demonstrating cardiovascular disease risk factors (7) and ischemic heart disease mortality (8). Most of these studies have been on Western populations, research in this area on Chinese older adult populations is limited.

The relationship between hypertension, diet, and exercise also exists in the older adult population. Despite the importance of nutrition for health, many older adults' food intake patterns are inconsistent with dietary guidelines. For example, studies from the United States, Europe, and Australia have demonstrated that older adults tend to have poor intakes of fruit, vegetables, dairy products, and whole grains, and a higher than desirable consumption of fatty food and fast food (9). Consequently, many older people fall short of achieving optimal nutrient intakes for good health. These unhealthy dietary patterns are associated with high risk of obesity (10) metabolic syndrome $(11,12)$, glucose abnormality (13), and cardiovascular disease (14).

Dietary patterns that conform with Western dietary guidelines are consistently associated with a lower risk of mortality $(9,15)$. A high consumption of vegetables and fruit is associated with decreased blood pressure in adults $(16,17)$, and higher than recommended food intake in fats and meat products is associated with being overweight and obese $(18,19)$, although this association is less clear in older adults. It has been established that a high risk for metabolic syndrome, diabetes, cardiovascular disease, and stroke can be mediated or modulated through effects on blood pressure, central body weight and obesity, fasting glucose, lipoprotein, and triglycerides (20). However, the relationship of dietary patterns to these biomarkers in Chinese older adults is unclear.

Several studies support the need for further research into dietary patterns and their effect on morbidity and mortality in older adults $(21,22)$. Dietary patterns have been shown to reflect 
variations in nutrient intake $(13,20,23)$. Individuals consume nutrients through a combination of food items; therefore, focusing on one specific dietary component is often inappropriate for determining the interactive effects among nutrients that might affect health outcomes in older adults. A focus on the complete dietary pattern of individuals is essential to capture complex diet behaviors and the potential relationship between nutrients and health outcomes $(13,14,23)$.

Some studies $(24,25)$ have found strong socioeconomic gradients in identified dietary patterns. For example, higher incomes and education levels are usually found to be associated with higher scores on the Healthy Eating Index, the Mediterranean Diet Score, and the Healthy Dietary Pattern (25). Other lifestyle factors such as smoking, alcohol consumption, and physical inactivity are reportedly related to both unhealthy dietary patterns (25) and mortality risks (26). The effect of cigarette smoking on weight status and blood pressure has been less studied in older people than it has been studied in adulthood, in which smoking has been found to be associated with increased arterial stiffness (27) and dyslipidemia (28-30). Physical inactivity is an independent risk factor for cardiovascular disease in older adults and has been found to be associated with high blood pressure $(31,32)$, high serum cholesterol, and lipid cholesterol, as well as glucose intolerance (33). The effects of smoking, alcohol consumption, and physical inactivity on the association between dietary patterns and cardiovascular risk factors have not been established in older people in China.

Given the lack of research on dietary patterns and cardiovascular risk factors in Chinese older adults, the aim of this study was to investigate the relationship between dietary habits and cardiovascular risk factors such as elevated blood pressure, metabolic syndrome, weight levels, and obesity in this population. The effects of socio-demographic factors, smoking, alcohol consumption, and physical inactivity on the relationship between dietary and cardiovascular risk factors were also examined. The following hypothesis was therefore proposed: there is relationship between dietary pattern and cardiovascular risk factors including elevated blood pressure, metabolic syndrome, and obesity in Chinese older adults.

\section{MATERIALS AND METHODS}

\section{STUDY DESIGN, SETTING, PARTICIPANTS, AND PROCEDURE}

Participants were randomly selected from people registered for a health check with the Changshu Centre for Disease Control and Prevention (CDC) in 2012. The study population consisted of Chinese older people aged 45-80 years in Changshu, Jiangsu Province. Changshu is situated in Southern China. Changshu has a gross domestic product (GDP) of over 4.80 trillion yuan (US\$759 billion), placing it second highest in a ranking of all Chinese provinces and municipal cities (34). Changshu in Jiangsu Province was selected as the study site because of its representation of small to medium size cities where most Chinese people reside. It also has well developed economic status in China, which has resulted in dramatic changes in the lifestyle, dietary, and disease patterns of its residents in recent years. Chronic, non-communicable diseases now account for an estimated $80 \%$ of total deaths and $70 \%$ of disability-adjusted life-years lost in Jiangsu province (35), making it exemplars of this change. To be included in the study participants had to be aged 50 or older, and live within $50 \mathrm{~km}$ of a metropolitan area. People who could not provide written consent to the study, had neurological impairments, severe mental illness, stroke, or heart failure were excluded from the study. Of the 750 eligible participants, $550(73.3 \%)$ gave written, informed consent for involvement in the study. This was sufficient to meet the minimum sample size requirement of 143 participants. Participants completed both the Food Frequency Questionnaire (FFQ) and biomarker tests, which were taken by qualified physicians.

\section{DIET- AND ACTIVITY-RELATED QUESTIONNAIRE}

The FFQ was developed as part of this study. The 24-h dietary recall method was used to collect food intake over two weekdays and one weekend on. Pictures were presented of 50 common foods in Chinese dishes/utensils in a range of sizes; the participants were asked to indicate portion/sizes consumed and how often they consumed the food (daily, weekly, or monthly). From this data, 34 frequently consumed items ( $90 \%$ of participants have chosen) were selected and grouped as follows: rice, flour, grains, beans, soybeans, soybean products, nuts including peanuts and sunflower seeds, deep-color vegetables, light-color vegetables, edible fungi and algae (such as mushrooms, agaric, and kelp), fruit, pork, poultry, seafood, beef/lamb/other red meat, organ meat, processed meat (such as ham, luncheon meat, canned meat), eggs, milk and yogurt, milk powder and cheese, confectionery, fast food, sugar, puffed food, and pastries. The amount of consumption of each food item was calculated individually per day per person.

Exploratory factor analysis extracted four dietary patterns from the 34-items. These patterns are best described as: (1) a "traditional food pattern" consisting of pork, poultry, fish and prawns, eggs, fruit, dark-color vegetables, light-color vegetables, rice, water, yogurt, fungi, peanuts, and sunflower seeds, pastries; (2) a "processed and fast food pattern," high in fats and sugars, consisting of meat snacks, puffed food, fast food, confectionery, carbonated drinks, and fruit and vegetable products; (3) a "soybean, grains, and flour food pattern" consisting of beans, grains, potatoes, soybeans, flours, and soybean products; and (4) a "milk products, other animal meats and animal organs pattern" consisting of animal liver, animal blood, other animal meats, commercial fruit and vegetable juices, cheese, fruit juices, fresh milk, and milk drinks. These patterns explained $17.48,9.52,5.57$, and $4.80 \%$ of the variance, respectively, comprising $37.37 \%$ of the total variance. The item milk powder had low factor loading. This item was deleted from the 34-item FFQ. Cronbach's alpha coefficients of 0.76 indicated a reasonable level of inter-item reliability for the questionnaire ( 0.69 for the traditional food pattern; 0.65 for the processed and fast food pattern; 0.73 for the soybeans, grains, and flour food pattern, and 0.63 for the milk products, animal liver, and other animal meat pattern, respectively). Pearson's correlation coefficients between the scores of the four patterns ranged from 0.13 to 0.27 , suggesting that these four food patterns are discrete and therefore independent of each other.

\section{ANTHROPOMETRY MEASURES}

The participants, wearing light, indoor clothing without shoes, were weighed to the nearest $0.1 \mathrm{~kg}$ and measured to the nearest $0.1 \mathrm{~cm}$ in height. BMI was calculated as weight (kilogram)/height 
$(\text { meter })^{2}$. The China Centre for Disease Control and Prevention (China CDC) and the Ministry of Health have adopted the World Diabetes Federation criteria (36) based on the BMI, and define "overweight" as $24.0-29.9 \mathrm{~kg} / \mathrm{m}^{2}$ and obesity as 30 and more than $30 \mathrm{~kg} / \mathrm{m}^{2}$.

Waist and hip girth (centimeter) were measured with an anthropometric tape over light clothing. Waist girth was measured at the minimum circumference between the iliac crest and the ribcage, and hip girth at the maximum width over the greater trochanters. The waist-hip ratio was then calculated as waist divided by hip measurements. Abdominal obesity was defined as waist circumference (WC), the maximum width over the greater abdomen, based on the World Diabetes Federation standard (36).

Systolic blood pressure (SBP) and diastolic blood pressure (DBP) were measured using an inflatable cuff wrapped around the upper arm (not the forearm or wrist) and attached to an electronic monitor that gave a digital readout of the BP (millimeter of mercury) and pulse. The average scores of SBP and DBP based on three measurements were used. The criteria for elevated BP are SBP $>130 \mathrm{mmHg}$ and DBP $>85 \mathrm{mmHg}$, based on the International Diabetes Federation (IDF) and China CDC criteria (36).

\section{MEASUREMENT OF METABOLIC ABNORMALITIES}

Metabolic syndrome was based on the updated IDF criteria (36). To be defined as having metabolic syndrome, a person must have central obesity (defined as $\mathrm{WC} \geq 90 \mathrm{~cm}$ for Chinese men and $\geq 80 \mathrm{~cm}$ for Chinese women, plus any two of the following four factors: (1) raised TG level: $\geq 150 \mathrm{mg} / \mathrm{dL}(1.7 \mathrm{mmol} / \mathrm{L})$; (2) reduced HDL cholesterol: $<40 \mathrm{mg} / \mathrm{dL}(1.03 \mathrm{mmol} / \mathrm{L})$ in males and $<50 \mathrm{mg} / \mathrm{dL}(1.29 \mathrm{mmol} / \mathrm{L})$ in females; (3) raised blood pressure: $\mathrm{SBP} \geq 130$ or diastolic $\mathrm{BP} \geq 85 \mathrm{mmHg}$; or (4) raised fasting plasma glucose $(\mathrm{FPG}) \geq 100 \mathrm{mg} / \mathrm{dL} \quad(5.6 \mathrm{mmol} / \mathrm{L})$. Dyslipidemia was defined as total high-density lipoprotein $<1.04 \mathrm{mmol} / \mathrm{L}$ for men and $<1.3$ for women, and/or triglycerides $(\mathrm{TG}) \geq 1.70 \mathrm{mmol} / \mathrm{L}$, according to the IDF definition (36).

\section{SAMPLE SIZE CALCULATION}

It was assumed that a standard deviation of SBP and DBP were about 14 and $8 \mathrm{mmHg}$, respectively. One hundred forty-three adults from a representative sample would be sufficient to provide an estimate of SBP and DBP of a population with $95 \% \mathrm{CI}$ around the mean of about \pm 120 . A large sample size up to 260 was required when precise measures including TG, cholesterol, blood glucose, were measured. As there were 550 adults were recruited into the study, the sample size is adequate for the current study.

\section{ETHICS APPROVAL}

Ethics approval for the research was obtained from Griffith University the Ethics Committee and the Changshu Centre for Disease Control and Prevention Research Board.

\section{STATISTICAL ANALYSIS}

Dietary patterns were derived from the factor analysis by adding all items that loaded highly on each factor. Pearson correlations were used to assess the independence of factors by analyzing the correction level between the pattern scores. Dietary pattern scores were categorized into tertiles with high, medium, and low levels to provide sensitive indicators of the food intake level to assess the association of a food pattern intake to the metabolic factors. Associations between dietary patterns, socio-demographic factors and food habits were investigated using chi-square analysis. Linear trends across tertiles of dietary pattern scores were calculated using a univariate linear modeling method. Means (and standard errors) of BMI, WC, SBP, and DBP were calculated according to tertiles of dietary pattern scores using the univariate linear modeling method and adjusted for age and sex. Only covariates associated with both the dietary patterns and health outcomes (income and exercise) were included in the adjusted models. The level of significance for $p$-values was set at 0.05 for all bivariate analyses and adjusted $p$-value for univariate linear modeling method.

\section{RESULTS}

Statistical assumption has been checked for all categorical variables for adequate cell number for Chi-Square test and continuous variables for Univariate Analysis of Variances. For all categorical variables, they all had adequate cell number to meet the requirement of Chi-Square tests. For all continuous variables, they all had normal distribution with skewness and Kurtosis test within -3 and +3 range.

\section{SAMPLE CHARACTERISTICS}

Table 1 shows, at baseline $(n=550), 58.6 \%$ of participants had "normal" weight, $34.3 \%$ were overweight, and $7 \%$ were obese. The prevalence of hypertension was $35.3 \%$. The prevalence of being overweight, obesity, and hypertension was similar to Chinese prevalence rates among older adults (37). In terms of age, $68.3 \%$ of participants were in the 50-64 age group, and $31.7 \%$ were aged 65-88. All participants were from a Han background. In terms of education level, $42.1 \%$ had a low level of primary school education, $40.1 \%$ had senior high school level, and $17.7 \%$ had a diploma or bachelor level qualification. Most participants were married $(89.4 \%)$ and retired $(95.7 \%)$. Nearly half $(44.4 \%)$ of the participants had an income level of $<20,000$ Chinese Yuan (RMB) per year, 37.1\% had income between RMB 20,000-39,999 per year, and only $18.5 \%$ had an income over RMB 40,000 per year. No differences were found between participants who participated in the biomedical assessment and those who did not in the demographic variables of age, gender, marital status, education, but there was a difference in income.

Table 2 demonstrates associations of dietary patterns with socio-demographic and behavioral characteristics. Only the soybeans, grains, and flour food pattern showed an association with income level; the high-income group was more likely to have a high intake for this pattern. Only the soybean, grains, and flour food pattern was associated with exercise; more hours of light exercise were evident in participants with this pattern. Age, gender, education level, marital status, and alcohol intake were not related to food intake patterns.

Table 3 demonstrates the association between dietary pattern and biomarkers. Income and light level exercise were associated with the soybeans, grains and flour dietary pattern; therefore, only these two confounders were included in the adjusted models in the analysis of the relationship between dietary pattern and health 
Table 1 | Demographic characteristics of participants.

\begin{tabular}{|c|c|c|c|c|}
\hline Variables & $\begin{array}{l}\text { Sample who did } \\
\text { not participate } \\
\text { biomedical tests } \\
N(\%)(n=200)\end{array}$ & $\begin{array}{l}\text { Study } \\
\text { sample } \\
N(\%) \\
(n=550)\end{array}$ & $\chi^{2}$ & $p$ \\
\hline \multicolumn{5}{|l|}{ Age } \\
\hline 50-64 & $136(68.3)$ & $122(63.5)$ & 2.69 & 0.11 \\
\hline 65-88 & $64(31.7)$ & 70 (36.5) & & \\
\hline \multicolumn{5}{|l|}{ Gender } \\
\hline Male & 39 (19.6) & $154(78.2)$ & 0.85 & 0.36 \\
\hline Female & $161(80.4)$ & 43 (21.8) & & \\
\hline \multicolumn{5}{|l|}{ Education } \\
\hline Primary schools & $84(42.1)$ & $61(31.0)$ & 2.10 & 0.09 \\
\hline Senior high schools & $80(40.1)$ & $91(46.2)$ & & \\
\hline TAFE and diploma & $27(13.7)$ & $29(14.7)$ & & \\
\hline Bachelor and above & $9(4.0)$ & $16(8.1)$ & & \\
\hline \multicolumn{5}{|l|}{ Occupation } \\
\hline Retired & $191(95.7)$ & 185 (93.9) & 2.57 & 0.11 \\
\hline Others & $9(4.3)$ & $12(6.1)$ & & \\
\hline \multicolumn{5}{|l|}{ Marital status } \\
\hline Married & $178(89.4)$ & $171(87.2)$ & 1.54 & 0.46 \\
\hline Widowed & $14(6.7)$ & $15(7.7)$ & & \\
\hline $\begin{array}{l}\text { Never married/ } \\
\text { divorced/separated }\end{array}$ & $8(3.9)$ & $10(5.1)$ & & \\
\hline \multicolumn{5}{|l|}{ Income } \\
\hline$<20,000 \mathrm{RMB}$ & $89(44.4)$ & 75 (38.9) & 10.06 & 0.01 \\
\hline 20,000-39,999 RMB & $74(37.1)$ & $68(35.2)$ & & \\
\hline 40,000 and more & $37(18.5)$ & 50 (25.9) & & \\
\hline \multicolumn{5}{|l|}{$\mathrm{BMI}$} \\
\hline Normal & $116(57.8)$ & $309(58.6)$ & 0.57 & 0.7 \\
\hline Overweight & 79 (39.7) & $181(34.3)$ & & \\
\hline Obesity & $5(2.5)$ & $37(7.0)$ & & \\
\hline \multicolumn{5}{|l|}{ Hypertension } \\
\hline Normal & $129(64.3)$ & $358(64.7)$ & 0.05 & 0.9 \\
\hline Hypertension & 71 (35.6) & $195(35.3)$ & & \\
\hline
\end{tabular}

Bold indicates $p<0.01$.

outcomes (Table 3). The level of significance for $p$-values was set at 0.02 for the analysis of the relationship between soybeans, grains, and flour dietary pattern and biomarkers due to the multiplicity of the analysis. The traditional food pattern that included fruit, vegetable, rice, and fish was significantly associated with SBP $(p=0.025)$. Among the three levels of food intake scores, participants with a moderate level of traditional food intake had lower SBP and cholesterol levels than those with a high level of traditional food intake.

The processed and fast food pattern was not significantly associated with any of the biomedical markers. The soybeans, grains, and flour food pattern was associated with WC $(p=0.04)$ and waist-hip ratio $(p=0.03)$, blood glucose $(p=0.03)$, and statistically significantly associated with triglycerides. Participants with a high level of soybeans, grains, and flour food intake had lower blood glucose $(p=0.03)$, WC $(p<0.001)$, and waist-hip ratio $(p=0.03)$ than those with a low level of this food intake pattern.
High intake levels of milk products, animal liver, and other animal meats were associated with increased BMI levels.

\section{DISCUSSION}

This study is the first to explore the association between dietary patterns and health outcomes in a population-based sample of Chinese older adults. The findings demonstrate that dietary patterns of Chinese older adults are associated with health outcomes. A moderate intake of "traditional Chinese food" is associated with reduced levels of SBP and cholesterol. A high intake of soybeans, grains, and flour is associated with a reduced level of metabolic factors, and a high intake of milk products, animal liver, and other animal meats is associated with increased BMI levels. There is no relationship between processed food and any of the cardiovascular disease risk factors. The hypothesis of a relationship between dietary pattern and cardiovascular risk is supported in three out of four diets identified in our factor analysis. It is difficult to compare these findings with earlier studies conducted in Western countries because Chinese older adults have substantially different food intake patterns. Regardless, the patterns identified demonstrate some similarities with previous work among older adults. For example, the fruit, vegetables, eggs, pork, and fish pattern as a "traditional" pattern has nutrient content similar to "vegetables, fruit, beef, lamb, fish, and grains" in other countries. The processed and fast food pattern is similar to the "Western food" pattern that includes meat pies, processed meats, pizza, chips, hamburgers, white bread, and sugar, while the soybeans, grains, and flour pattern is similar to the "modern" food pattern. Finally, the dairy, animal liver, and other animal meat food pattern is similar to the traditional Western food pattern (38).

The validity of the dietary patterns was assessed by examining the association between food patterns and biomarkers. Several methodological issues strengthen confidence in the questionnaire. The screening tool was developed based on 24-h food intake data in the previous month and is quantitative, thus minimizing errors in memory and computation. Notable correlations with biomarkers of nutritional status provide support for the questionnaire, because measurement error in the questionnaire is not likely to be correlated to measurement error in the assessment of biomarkers. Bailey et al. (23) and van Staveren et al. (39) have indicated favorable intakes of micronutrients and biomarkers among older adults who consume foods rich in fruit and grains. Although pattern differences across groups are likely, the argument for generalizability is that the patterns detected using the questionnaire were similar to those found in more diverse samples, with significantly more comprehensive dietary assessment methods.

This study found that only the soybean, grains, and flour dietary pattern was associated with measures of income, which is consistent with research carried out in various populations using different indices $(13,14,31)$. The general observed socioeconomic nutritional gradient can be mediated by food costs, meaning that the lowest cost diets, mainly consumed by the lowest socioeconomic positions, are generally unhealthy. People who consume higher levels of soybean products, grains, and flour food reported higher incomes, suggesting that these foods are viewed by this group as high quality and affordable. 


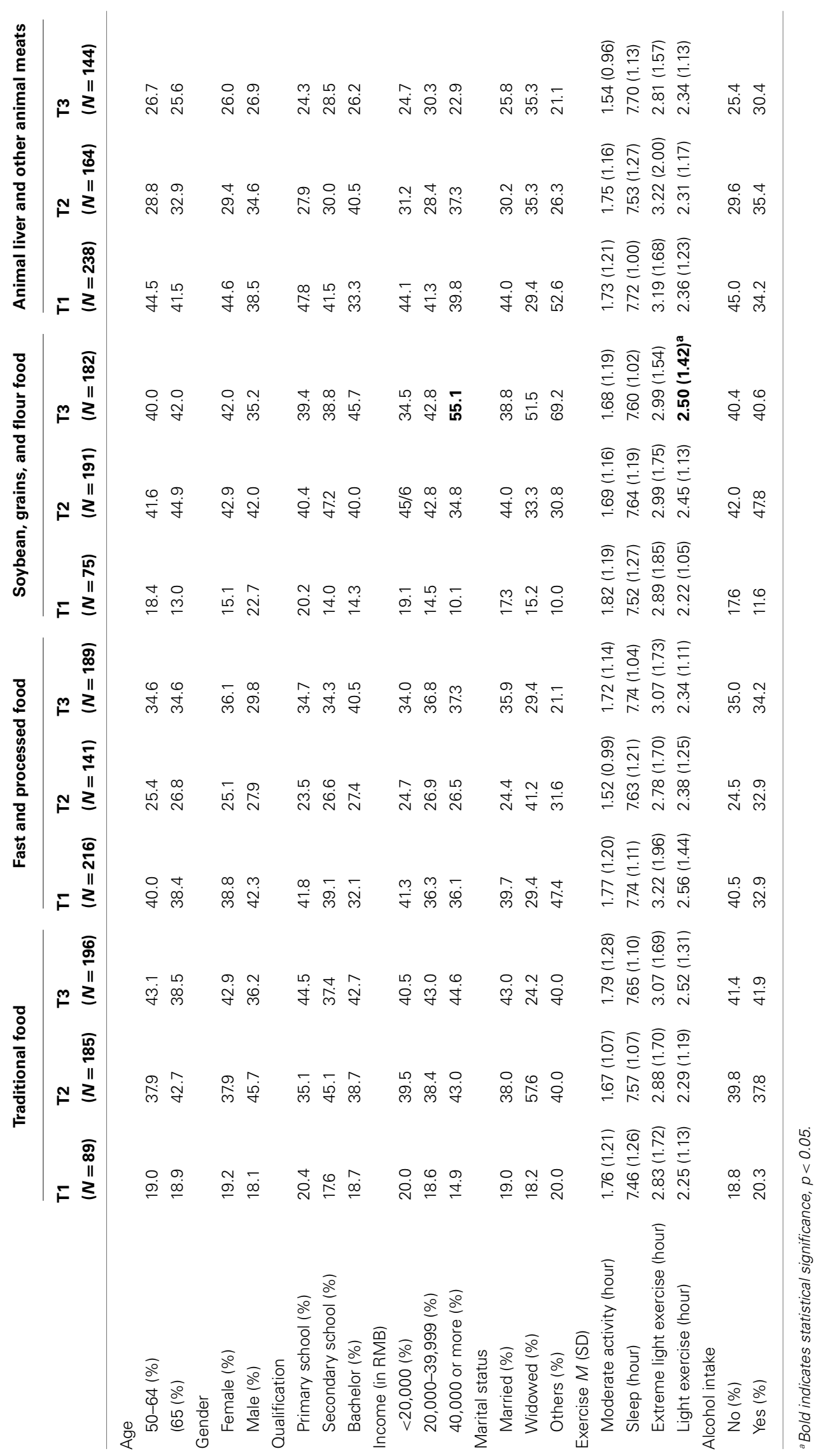




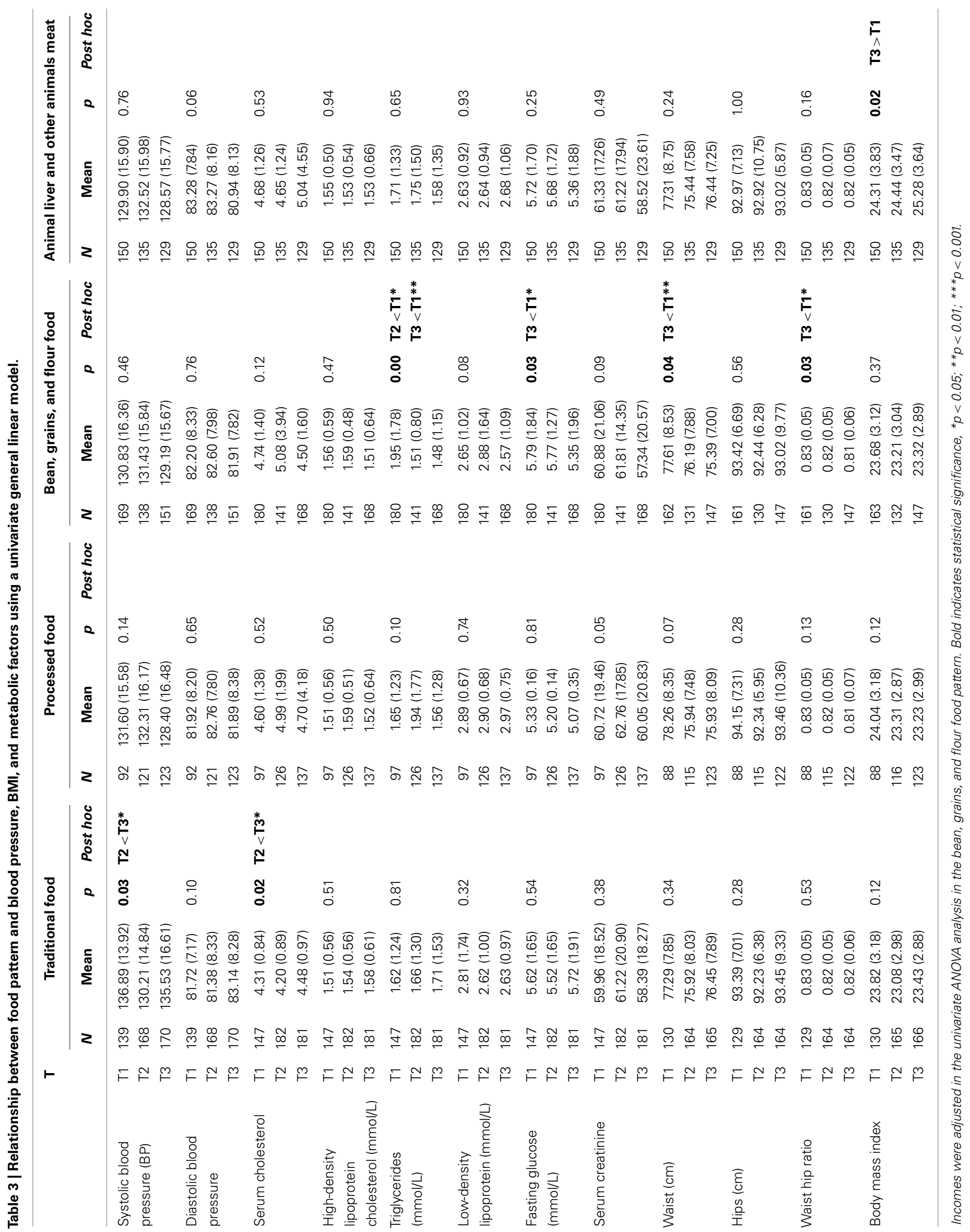


Participants with moderate intakes of traditional Chinese food, including the fruit, vegetables, pork, chicken, rice, and fish pattern, had lower SBP and serum cholesterol levels, but no other biomarkers. No previously published literature explains this outcome, but it may be that the nutritional characteristics of the traditional Chinese diet (for instance) positively affect blood pressure levels. These characteristics include moderate levels of fruit, vegetables, and protein, possibly leading to dietary-induced lowered blood pressure level (17). The prevalence of hypertension and stroke is high for older Chinese adults (17), and the positive association between healthy food intake and blood pressure is well documented. Considering that the moderate intake of traditional Chinese foods is the dominant food pattern, this food intake pattern may be one of the causes of decreased blood pressure levels. Processed and fast food was not related to any of the biomedical markers. It is speculated that the low intake of processed and fast food may account for its minimal-level effect on metabolism, and may account for the fact that the prevalence of coronary heart disease among Chinese is lower than it is for Caucasians (40).

The soybean, grains, and flour food dietary pattern, which was correlated with a lower WC and waist-hip ratio, has also been associated with several biomarkers for reduced cardiovascular risk factors, including a reduced level of serum lipoproteins and blood glucose. The high consumption of soybean products may contain more phytoestrogen, which has protective effects against heart disease (13). The finding that consumers of the soybean, grains, and flour pattern had lower blood glucose, triglycerides, WC, and waist-hip ratios is consistent with previous research (41) and, therefore, provides additional evidence that a dietary intake of these foods may reduce the risk of obesity, metabolic syndrome, and diabetes in Chinese older adults.

Components of the milk products, animal food dietary pattern included animal liver and other animal foods. Higher scores on this pattern likely accompany greater consumption of fat, especially saturated fat, which may increase the risk of diabetes, metabolic syndrome, and hypertension $(16,42)$. The Western dietary pattern is characterized by frequent red meat intake, which increases the risk of cardiovascular disease $(16,42)$. Consistent with these findings, the present study found that the milk products and animal food dietary pattern was significantly associated with an increased level of BMI. Dietary habit change involving less consumption of other animal meats may offer avenues for minimizing high BMI levels.

Our detailed analysis of dietary patterns reflects intakes of nutrients important for the prevention of factors associated with high SBP, metabolic abnormalities, and cardiovascular diseaserelated biomedical risk factors. This highlights that the dietary patterns measure underlying differences in nutrient intakes, capture biologically relevant exposures, and describe eating patterns. Overall, Chinese foods have less fat than European diets. Consuming a variety of food, including soybeans, grains, and flour food, milk products, and a moderate level of other animal meats therefore may be complementary to the moderate-level traditional food intake in China. This is evident from the association of biomarkers with the two main food patterns that are protective against high blood pressure, high metabolic abnormality level, and high levels of WC and waist-hip ratio.
The moderate "traditional Chinese food" intake is related to a reduced chance of having high blood pressure compared with that of the high-level "traditional Chinese food" intake. Intake levels in particular food patterns are related to different metabolic outcomes as follows: (a) a high level of soybean product, grain, and flour food pattern is related to a reduced chance of being overweight or obese, and having a metabolic abnormality and glucose intolerance; and (b) a high level of dairy and other cooked animal meats is related to an increased risk of being overweight and obesity.

The results arising from these food intake patterns suggest that moderate intakes of "traditional Chinese food," high-level intakes of soybean products and grains, and low-level intakes of milk products and animal meats are protective against metabolic syndrome, high blood pressure, elevated triglycerides, and obesity, all of which are key risk factors for cardiovascular disease and glucose intolerance, a key risk factor for Type 2 diabetes.

A limitation of this study is the high proportion of older adults ( $n=200 ; 26.7 \%$ ) who did not complete a biomarker test and were thus excluded from the analysis; however, there were no differences between people who participated in the study and those who did not participate. In addition, the effect of a moderate intake level of traditional Chinese food is only speculative due to the nature of the cross-sectional study data and further nutrient data analysis is required to confirm this result. Further study using prospective cohort or intervention study should be used to confirm the association between dietary patterns and blood pressure and metabolic factors. Although the sample size is large with 550 people consented to the study, no all participants completed questions and biomedical assessments, so the number of participants varied for assessment items. The bias caused by these missing data should be taken into consideration when the conclusion is made and when study is generalized to other regions in China.

In conclusion, the present results indicate that a dietary pattern characterized by frequent consumption of a moderate level of "traditional Chinese food," high-level intake of soybean products and dairy products, combined with a low intake of animal meat products, may be associated with a reduced risk of impaired glucose tolerance, reduced SBP in Chinese older adults and BMI. Although these findings need to be confirmed by prospective studies, interventions to change dietary patterns should be considered to decrease cardiovascular disease risk among Chinese older adults, whose consumption of dairy food and fruit is currently low.

\section{RELEVANCE TO CLINICAL PRACTICE}

We recommend that strategies targeting relevant dietary intake factors be implemented in the population of adults aged 50 and above in China to manage cardiovascular disease risk factors. Health professionals such as nurses may be best suited to providing such interventions given their integral role of providing advice to people with unhealthy food intake. Cardiovascular disease management should be implemented in community medical centers close to the population, and focus service delivery on measures to assist patients to consume a moderate level of "traditional Chinese food," a high level of soybean products and dairy products, and a low intake of animal meat products. 


\section{AUTHORS CONTRIBUTION}

Dr. Jing Sun: conceptualized and designed the study, did field work, prepared and drafted manuscript paper, analyzed data, and revised the manuscript. Professor Nicholas Buys: designed the study, reviewed, and revised the manuscript. Dr. Shuying Shen: designed the food frequency questionnaire, and proofed the manuscript.

\section{ACKNOWLEDGMENTS}

The project was jointly funded by Griffith University in Australia and Changshu Center for Disease Control and Prevention, and a support was sought to Changshu Center for Disease Control and Prevention and local health government in Changshu, China in relation to access of health check data during the participant recruitment stage and completion of data collection work.

\section{REFERENCES}

1. Gu D, Chen J, Wu X, Duan X, Jones DW, Huang J, et al. Prehypertension and risk of cardiovascular disease in Chinese adults. J Hypertens (2009) 27:721-9. doi:10.1097/HJH.0b013e328323ad89

2. Gu D, Reynolds K, Wu X, Chen J, Duan X, Muntner P, et al. Prevalence, awareness, treatment, and control of hypertension in China. Hypertension (2002) 40:920-7. doi:10.1161/01.HYP.0000040263.94619.D5

3. Perkovic V, Huxley R, Wu Y, Prabhakaran D, MacMahon S. The burden of blood pressure-related disease: a neglected priority for global health. Hypertension (2007) 50:991-7. doi:10.1161/HYPERTENSIONAHA.107.095497

4. Geleijnse JM, Kok FJ, Grobbee DE. Blood pressure response to changes in sodium and potassium intake: a metaregression analysis of randomised trials. J Hum Hypertens (2003) 17:471-80. doi:10.1038/sj.jhh.1001575

5. Marshall AL, Booth ML, Bauman AE. Promoting physical activity in Australian general practice: a randomised trial of health promotion advice versus hypertension managment. Patient Educ Couns (2005) 56:283-90. doi:10.1016/j.pec. 2004.03.002

6. Zhou BF, Stamler J, Dennis B, Moag-Stahlberg A, Okuda N, Robertson C, et al. Nutrient intakes of middle-aged men and women in China, Japan, United Kingdom, and United States in the late 1990s: the INTERMAP Study. J Hum Hypertens (2003) 17:623-30. doi:10.1038/sj.jhh.1001605

7. Hellénius ML, de Faire U, Berglund B, Hamsten A, Krakau I. Diet and exercise are equally effective in reducing risk for cardiovascular disease. Results of a randomized controlled study in men with slightly to moderately raised cardiovascular risk factors. Atherosclerosis (1993) 103(1):81-91. doi:10.1016/00219150(93)90042-S

8. Ellingsen I, Hjermann I, Abdelnoor M, Hjerkinn EM, Tonstad S. Dietary and antismoking advice and ischemic heart disease mortality in men with normal or high fasting triacylglycerol concentrations: a 23-y follow-up study. Am J Clin Nutr (2003) 78(5):935-40.

9. Dwyer T, Hetzel BS. A comparison of trends of coronary heart disease mortality in Australia, USA and England and Wales with reference to three major risk factors-hypertension, cigarette smoking and diet. Int J Epidemiol (1980) 9(1):65-71. doi:10.1093/ije/9.1.65

10. Quatromani PA, Copenhafer DL, D'Agostino RB, Millen BE. Dietary patterns predict the development of overweight in woman: the Framingham Nutrition Studies. J Am Diet Assoc (2002) 102(9):1239-46. doi:10.1016/S0002-8223(02) 90275-0

11. Sonnenberg L, Pencina M, Kimokoti R, Quatromoni P, Nam BH, D’Agostino $\mathrm{R}$, et al. Dietary patterns and the metabolic syndrome in obese and non-obese Framingham women. Obesity (Silver Spring) (2005) 13(1):153-62. doi:10.1038/ oby. 2005.20

12. Hydrie MZI, Basit A, Shera AS, Hakeem R, Hussain A. Dietary patterns associated with risk for metabolic syndrome in urban community of Karachi defined by cluster analysis. Pakistan J Nutr (2010) 9(1):93-9. doi:10.3923/pjn.2010.93.99

13. Mizoue T, Yamaji T, Tabata S, Yamaguchi K, Ogawa S, Mineshita M, et al. Dietary patterns and glucose tolerance abnormalities in Japanese men. J Nutr (2006) 136(5):1352-8.

14. Schulze M, Hu F. Dietary patterns and risk of hypertension, type 2 diabetes mellitus, and coronary heart disease. Curr Atheroscler Rep (2002) 4(6):462-7. doi:10.1007/s11883-002-0051-1
15. Russell J, Flood V, Rochtchina E, Gopinath B, Allman-Farinelli M, Bauman A, et al. Adherence to dietary guidelines and 15-year risk of all-cause mortality. $\mathrm{Br}$ J Nutr (2013) 109(3):547-55. doi:10.1017/S0007114512001377

16. Hsiao PY, Mitchell DC, Coffman DL, Allman RM, Locher JL, Sawyer P, et al. Dietary patterns and diet quality among diverse older adults: the University of Alabama at Birmingham Study of Aging. J Nutr Health Aging (2013) 17(1):19-25. doi:10.1007/s12603-012-0082-4

17. Viscogliosi G, Cipriani E, Liguori ML, Marigliano B, Saliola M, Ettorre E, et al. Mediterranean dietary pattern adherence: associations with prediabetes, metabolic syndrome, and related microinflammation. Metab Syndr Relat Disord (2013) 11(3):210-6. doi:10.1089/met.2012.0168

18. Esmaillzadeh A, Azadbakht L. Major dietary patterns in relation to general obesity and central adiposity among Iranian women. J Nutr (2008) 138(2):358-63.

19. Flores M, Macias N, Rivera M, Lozada A, Barquera S, Rivera-Dommarco J, et al. Dietary patterns in Mexican adults are associated with risk of being overweight or obese. J Nutr (2010) 140(10):1869-73. doi:10.3945/jn.110.121533

20. Hu FB, Manson JE, Willett WC. Types of dietary fat and risk of coronary heart disease: a critical review. J Am Coll Nutr (2001) 20(1):5-19. doi:10.1080/ 07315724.2001.10719008

21. Menotti A, Alberti-Fidanza A, Fidanza F, Lanti M, Fruttini D. Factor analysis in the identification of dietary patterns and their predictive role in morbid and fatal events. Public Health Nutr (2012) 15(7):1232-9. doi:10.1017/ S1368980011003235

22. Waijers PM, Ocké MC, van Rossum CT, Peeters PH, Bamia C, Chloptsios Y, et al. Dietary patterns and survival in older Dutch women. Am J Clin Nutr (2006) 83(5):1170-6.

23. Bailey RL, Mitchell DC, Miller CK, Still CD, Jensen GL, Tucker KL, et al. A dietary screening questionnaire identifies dietary patterns in older adults. J Nutr (2007) 137(2):421-6.

24. Giuli C, Papa R, Mocchegiani E, Marcellini F. Dietary habits and ageing in a sample of italian older people. J Nutr Health Aging (2012) 16(10):875-9. doi:10.1007/s12603-012-0080-6

25. Mullie P, Clarys P, Hulens M, Vansant G. Dietary patterns and socioeconomic position. Eur J Clin Nutr (2010) 64(3):231-8. doi:10.1038/ejcn.2009.145

26. Héroux M, Janssen I, Lam M, Lee DC, Hebert JR, Sui X, et al. Dietary patterns and the risk of mortality: impact of cardiorespiratory fitness. Int J Epidemiol (2010) 39(1):197-209. doi:10.1093/ije/dyp191

27. Hata K, Nakagawa T, Mizuno M, Yanagi N, Kitamura H, Hayashi T, et al. Relationship between smoking and a new index of arterial stiffness, the cardio-ankle vascular index, in male workers: a cross-sectional study. Tob Induc Dis (2012) 10(1):11. doi:10.1186/1617-9625-10-11

28. Chang SA. Smoking and type 2 diabetes mellitus. Diabetes Metab J (2012) 36(6):399-403. doi:10.4093/dmj.2012.36.6.399

29. Patja K, Jousilahti P, Hu G, Valle T, Qiao Q, Tuomilehto J. Effects of smoking, obesity and physical activity on the risk of type 2 diabetes in middle-aged Finnish men and women. J Intern Med (2005) 258(4):356-62. doi:10.1111/j.1365-2796. 2005.01545.x

30. Sairenchi T, Iso H, Nishimura A, Hosoda T, Irie F, Saito Y, et al. Cigarette smoking and risk of type 2 diabetes mellitus among middle-aged and elderly Japanese men and women. Am J Epidemiol (2004) 160(2):158-62. doi:10.1093/ aje/kwh183

31. Appleton SL, Neo C, Hill CL, Douglas KA, Adams RJ. Untreated hypertension: prevalence and patient factors and beliefs associated with undertreatment in a population sample. J Hum Hypertens (2012) 27(7):453-62. doi:10.1038/jhh.2012.62

32. Vagetti GC, Barbosa Filho VC, Moreira NB, de Oliveira V, Mazzardo O, de Campos W. The prevalence and correlates of meeting the current physical activity for health guidelines in older people: a cross-sectional study in Brazilian women. Arch Gerontol Geriatr (2013) 56(3):492-500. doi:10.1016/j.archger. 2012.12.003

33. Baan CA, Stolk RP, Grobbee DE, Witteman JC, Feskens EJ. Physical activity in elderly subjects with impaired glucose tolerance and newly diagnosed diabetes mellitus. Am J Epidemiol (1999) 149(3):219-27. doi:10.1093/oxfordjournals.aje. a009795

34. Staff Reporter. Jiangsu Unchained: Top in Per Capita GDP, Guangdong in its Sights. Tai Pei: Economy (2013).

35. Wang X, Lu M, Qian J, Yang Y, Li S, Lu D, et al. Rationales, design and recruitment of the Taizhou Longitudinal Study. BMC Public Health (2009) 9:223. doi:10.1186/1471-2458-9-223 
36. Wu A, Kong N, de Leon F. An alarmingly high prevalence of diabetic nephropathy in Asian type 2 diabetic patients: the MicroAlbuminuria Prevalence (MAP) Study. Diabetologia (2005) 48:17-26. doi:10.1007/s00125-004-1599-9

37. He Y, Li Y, Lai J, Wang D, Zhang J, Fu P, et al. Dietary patterns as compared with physical activity in relation to metabolic syndrome among Chinese adults. Nutr Metab Cardiovasc Dis (2013) 23(10):920-8. doi:10.1016/j.numecd.2012.09.001

38. Bouillon K, Batty GD, Hamer M, Sabia S, Shipley MJ, Britton A, et al. Cardiovascular disease risk scores in identifying future frailty: the Whitehall II prospective cohort study. Heart (2013) 99(10):737-42. doi:10.1136/heartjnl2012-302922

39. van Staveren WA, Steijns JM, de Groot LC. Dairy products as essential contributors of (micro-) nutrients in reference food patterns: an outline for elderly people. J Am Coll Nutr (2008) 27(6):747S-54S. doi:10.1080/07315724.2008. 10719753

40. Keyou KG. The transition of Chinese dietary guidelines and food guide pagoda. Asia Pac J Clin Nutr (2011) 20(3):439-46.

41. Guevara-Cruz M, Tovar AR, Aguilar-Salinas CA, Medina-Vera I, Gil-Zenteno L, Hernández-Viveros I, et al. A dietary pattern including nopal, chia seed, soy protein, and oat reduces serum triglycerides and glucose intolerance in patients with metabolic syndrome. J Nutr (2012) 142(1):64-9. doi:10.3945/jn. 111.147447
42. Hu FB, Rimm EB, Stampfer MJ, Ascherio A, Spiegelman D, Willett WC. Prospective study of major dietary patterns and risk of coronary heart disease in men. Am J Clin Nutr (2000) 72:912-21.

Conflict of Interest Statement: The authors declare that the research was conducted in the absence of any commercial or financial relationships that could be construed as a potential conflict of interest.

Received: 10 September 2013; accepted: 20 October 2013; published online: 01 November 2013.

Citation: Sun J, Buys $N$ and Shen S (2013) Dietary patterns and cardiovascular disease-related risks in Chinese older adults. Front. Public Health 1:48. doi: 10.3389/fpubh.2013.00048

This article was submitted to Epidemiology, a section of the journal Frontiers in Public Health.

Copyright (c) 2013 Sun, Buys and Shen. This is an open-access article distributed under the terms of the Creative Commons Attribution License (CC BY). The use, distribution or reproduction in other forums is permitted, provided the original author $(s)$ or licensor are credited and that the original publication in this journal is cited, in accordance with accepted academic practice. No use, distribution or reproduction is permitted which does not comply with these terms. 